\title{
Solução do Problema de Deflexão de Vigas com o Método das Diferenças Finitas
}

\author{
Gabrielle Piezzoti Oliveira* \\ Coordenação de Ciência da Computação, COCIC, UTFPR \\ 80230-901, Campus Campo Mourão, Campo Mourão, PR \\ E-mail: gabrielle793@hotmail.com \\ Viviane Colucci Adilandri Mércio Lobeiro \\ Departamento de Matemática, DAMAT, UTFPR \\ 80230-901, Campus Campo Mourão, Campo Mourão, PR \\ E-mail: colucci@utfpr.edu.br, alobeiro@utfpr.edu.br

\section{Clicia Geovana Alves Pereira} \\ Departamento de Ciências, UEM \\ 87360-000, Campus Goioerê, Goioerê, PR \\ E-mail: cgapereira2@uem.br
}

\section{$\underline{\text { RESUMO }}$}

Este trabalho trata da obtenção das soluções analítica e numérica de um Problema de Valor de Contorno (PVC) para o problema de Deflexão de Vigas.

Em particular, considera-se uma viga bi-apoiada de seção transversal retangular, de modo que as extremidades não sofrem deflexão, com uma carga uniformemente distribuída sobre ela, como apresentada na Figura 1.

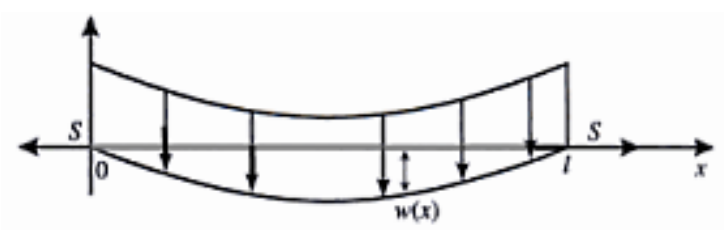

Figura 1: Viga com extremidades apoiadas, sujeita a carga uniforme

A Equação Diferencial Ordinária Linear que aproxima o caso à situação física é dada por:

$$
\frac{d^{2} w}{d x^{2}}=\frac{S}{E I} w+\frac{q x}{2 E I}(x-l)
$$

onde $w(x)$ é a deflexão a uma distância $x$ a partir da extremidade esquerda da viga, $l$ representa o comprimento da viga, $q$ a intensidade de carga uniforme a que está submetida, $E$ o módulo da elasticidade, $S$ o esforço nas extremidades e $I$ representa o momento de inércia central.

Como não há deflexão nas extremidades da viga, vale as condições: $w(0)=0$ e $w(l)=0$.

Para um estudo de caso, considera-se uma viga de aço com as seguintes características:

$$
l=120 \mathrm{pol}, \quad q=100 \mathrm{lb} / \mathrm{pé}=\frac{100}{12} \mathrm{lb} / \mathrm{pol}, \quad E=3,0 \times 10^{7} \mathrm{lb} / \mathrm{pol}^{2}, S=1000 \mathrm{lb} \text { e } I=625 \mathrm{pol}^{4} .
$$

\footnotetext{
*bolsista de Iniciação Científica PICME, CNPq/Capes
} 
Obtém-se então o Problema de Valor de Contorno (PVC)

$$
\left|\begin{array}{l}
\frac{d^{2} w}{d x^{2}}-\frac{1.0 \cdot 10^{-4}}{1875} w=\frac{1.0 \cdot 10^{-9}}{4.5}\left(x^{2}-120 x\right) \\
w(0)=0 \text { e } w(120)=0
\end{array}\right|
$$

cuja solução analítica é dada por

$$
w(x)=c_{1} e^{m x}+c_{2} e^{-m x}+c_{3} x^{2}+c_{4} x+c_{5},
$$

onde $c_{1}=\frac{\left(\frac{5}{32} \cdot 10^{6}\right)\left(1-e^{-2 \cdot \sqrt{3} / 125}\right)}{e^{2 \cdot \sqrt{3} / 125}-e^{-2 \cdot \sqrt{3} / 125}}, c_{2}=\frac{\left(\frac{5}{32} \cdot 10^{6}\right)\left(e^{2 \cdot \sqrt{3} / 125}-1\right)}{e^{2 \cdot \sqrt{3} / 125}-e^{-2 \cdot \sqrt{3} / 125}}, m=\frac{\sqrt{3}}{7500}, c_{3}=\frac{-1}{240}, c_{4}=\frac{1}{2}$ e $c_{5}=-\frac{5}{32} \cdot 10^{6}$.

Para obter a solução numérica do PVC, foi aplicado o método das Diferenças Finitas, substituindo-se as derivadas da equação pelas fórmulas de diferenças centrais [1]

$$
\mid \begin{aligned}
& -w_{i-1}+\left(2+h^{2}\left(\frac{1.0 \cdot 10^{-4}}{1875}\right)\right) w_{i}-w_{i+1}=-h^{2}\left(\frac{1.0 \cdot 10^{-9}}{4.5}\right)\left(x^{2}-120 x\right) \\
& w_{0}=0 \text { e } w_{N+1}=0
\end{aligned},
$$

obtendo um sistema linear de equações, para $i$ variando de 1 até $N$.

Dividiu-se o intervalo [0,120] (comprimento da viga) em 1200 subintervalos iguais, cujas extremidades são os pontos da malha $x_{i}=i h$, para $i=0, \ldots, 1201$, sendo $h=0,1$ (passo), para os quais busca-se a aproximação $w_{i}$. Quanto mais discretizado o intervalo, mais precisas são as aproximações. No entanto, tendo em vista o custo computacional e que a discretização aplicada foi suficiente para se obter um resultado satisfatório, não foi necessário um passo menor.

A forma matricial do sistema gerado trabalha com uma matriz de coeficientes tridiagonal $1199 \times 1199$.

O código em MATLAB a seguir implementa o método e resolve o sistema.

Código 1: Solução Numérica de um PVC com o Método de Diferenças Finitas

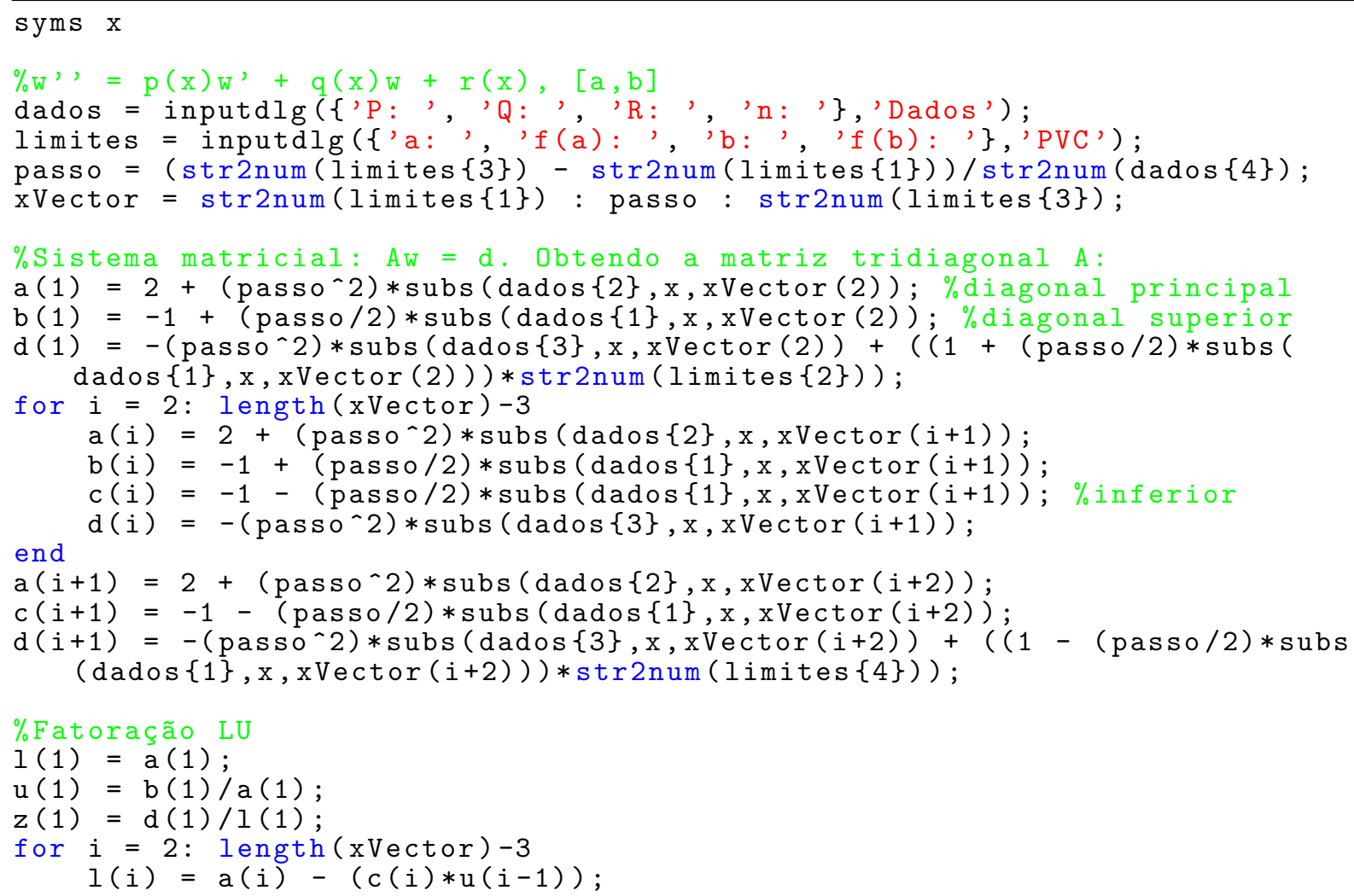


end

$u(i)=b(i) / l(i)$;

$z(i)=(d(i)-(c(i) * z(i-1))) / l(i)$;

$l(i+1)=a(i+1)-(c(i+1) * u(i)) ;$

$z(i+1)=(d(i+1)-(c(i+1) * z(i))) / 1(i+1) ;$

$\%$ Resolvendo o sistema

$\mathrm{W}(1)=\operatorname{str} 2 \operatorname{num}(1 \mathrm{imites}\{2\}) ;$

$\mathrm{W}($ length $(\mathrm{xVector}))=\operatorname{str} 2 \operatorname{num}(\operatorname{limites}\{4\}) ;$

$\mathrm{w}($ length $(\mathrm{xVector})-1)=\mathrm{z}($ length $(\mathrm{xVector})-2)$;

for $i=$ length (xVector) $-2:-1: 2$

$w(i)=z(i-1)-(u(i-1) * w(i+1)) ;$

end

A Figura 2 apresenta os gráficos de ambas as soluções.

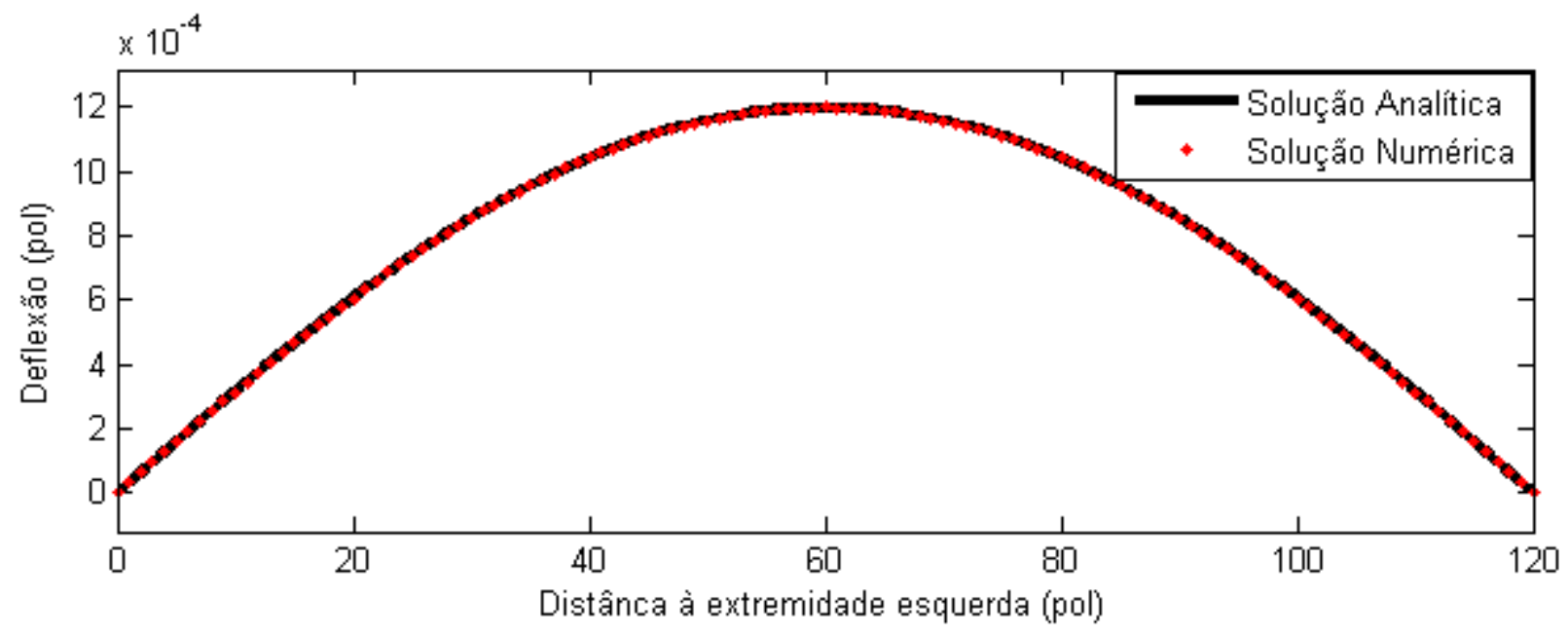

Figura 2: Soluções Gráficas

Com as soluções gráficas fica visível a proximidade entre os resultados. A Tabela a seguir exemplifica alguns dos valores encontrados, também para facilitar a comparação entre as soluções.

\begin{tabular}{ccccc}
\hline$x_{i}$ & $w\left(x_{i}\right)$ & $w_{i}$ & Erro absoluto & Erro percentual \\
\hline 0 & 0 & 0 & 0 & 0 \\
20 & 0.0006073605 & 0.0006073609 & 0.0000000004 & 0.0000658587 \\
40 & 0.0010428818 & 0.0010428824 & 0.0000000006 & 0.0000575329 \\
60 & 0.0011999063 & 0.0011999070 & 0.0000000007 & 0.0000583379 \\
80 & 0.0010428818 & 0.0010428824 & 0.0000000006 & 0.0000575329 \\
100 & 0.0006073605 & 0.0006073609 & 0.0000000004 & 0.0000658587 \\
120 & 0 & 0 & 0 & 0 \\
\hline
\end{tabular}

Tabela 1: Resultados e Erros

Pode-se concluir, pela ordem de magnitude dos erros, que ficou entre $0.00005 \%$ e $0.00007 \%$, a eficácia do método numérico.

Palavras-chave: Equação Diferencial Ordinária, Solução Numérica, Diferenças Finitas, Deflexão de Vigas

\section{Referências}

[1] R.L. Burden, J.D. Faires, Análise Numérica, Cengage Learning, 2008. 